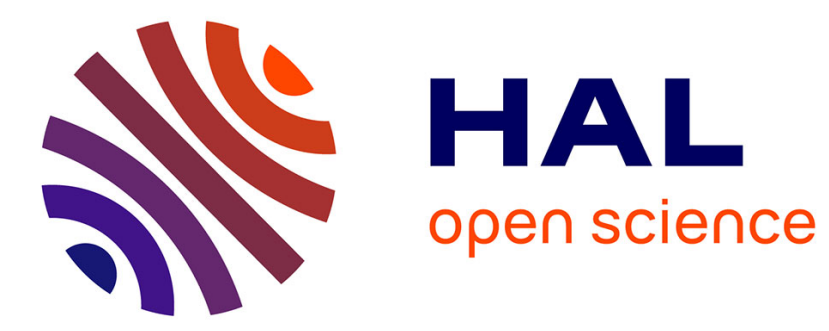

\title{
As many antipodes as vertices on convex polyhedra
}

J. Rouyer, Tewfik Sari

\section{To cite this version:}

J. Rouyer, Tewfik Sari. As many antipodes as vertices on convex polyhedra. Advances in Geometry, 2012, 12 (1), pp.43 -61. 10.1515/advgeom.2011.030 . hal-00777554

\section{HAL Id: hal-00777554 https://hal.science/hal-00777554}

Submitted on 17 Jan 2013

HAL is a multi-disciplinary open access archive for the deposit and dissemination of scientific research documents, whether they are published or not. The documents may come from teaching and research institutions in France or abroad, or from public or private research centers.
L'archive ouverte pluridisciplinaire HAL, est destinée au dépôt et à la diffusion de documents scientifiques de niveau recherche, publiés ou non, émanant des établissements d'enseignement et de recherche français ou étrangers, des laboratoires publics ou privés. 


\title{
As many antipodes as vertices on convex polyhedra
}

\author{
Joël Rouyer Tewfik Sari
}

June 14, 2010

\begin{abstract}
An earlier result states that, on the surface of a convex polyhedron with $n$ vertices endowed with its intrinsic metric, a point cannot have more than $n$ antipodes (farthest points). In this paper we produce examples of polyhedra with $n$ vertices, on which some suitable point admits exactly $n$ antipodes. We also proved that, for any positive number $d \leq 1$, there exist (in the closure of the set of these polyhedra) some convex surfaces on which some point have a set of antipodes of Hausdorff dimension $d$.
\end{abstract}

MSC (2000): 52B10, 53C45.

\section{Introduction}

Geographers define the antipode of some point $p$ belonging to a sphere, or to a slightly flattened ellipsoid of revolution - namely the Earth - as the point diametrically opposite to $p$. It is also the farthest point from $p$. Mathematicians will prefer this latter definition which advantageously remains valid in any compact metric spaces, although antipodes are no longer necessarily unique.

Note that the equivalence between the above two definitions is far from obvious in the case of a flattened ellipsoid [13], and false for many surfaces, as a stretched ellipsoid of revolution [14], or the surface of a centrally symmetric polyhedron [11]. Indeed, the equivalence occurs so rarely that H. Steinhaus formulated the conjecture that the sphere is the only convex surface on which the antipodal map is a single-valued involution [7]. The conjecture was not disproved before C. Vîlcu's discovery of some family of counter-examples, including the case of a flattened ellipsoid of revolution [13].

We recall that a convex surface $\mathcal{S}$ is the boundary of some convex body (i.e. compact convex subset with nonempty interior) of $\mathbb{R}^{3}$ endowed with its so-called intrinsic metric: the distance between two points is the length of the shortest curve of $\mathcal{S}$ which joins them.

We said previously that a point may have more than one antipode. It is rather obvious if you consider a long cigar shaped surface. Points near an 
extremity surely have antipodes near the other one, and by the middle, some points must have at least one antipode near each extremity. However the set of points admitting more than one antipode is small [17]. This fact has been investigated beyond the frame of convex surfaces, though the notion of smallness depends on the studied case $[8,9]$.

A point may also have infinitely many antipodes. As an example, if you identify on a round sphere the points which have the same latitude, and longitudes equal modulo $\pi$, on the resulting surface (which is actually isometric to some convex one, by virtue of Alexandrov's gluing theorem [2, p. 315-20]) a point of the equator admits a whole meridian as set of antipodes.

Nevertheless the set of antipodes of some point $p$ of a convex surface cannot be too big. Tudor Zamfirescu proved that its Hausdorff dimension is at most 1 , and the quotient of its length (1-dimensional Hausdorff measure) and the distance between $p$ and one of its antipodes is never more than $\pi$. Further, the set of antipodes of a point is always homeomorphic to some compact subset of the interval $[0,1]$. No more could be said - topologically speaking - about it, for each compact subset of $[0,1]$ can be realized as the set of antipodes of some suitable point of some suitable convex surface $[15,16]$.

Special attention was paid to the case of polyhedral surfaces. It is proved in [11] - among other facts - that no point on any polyhedron with $n$ vertices can have more than $n$ antipodes (Theorem 7). The aim of this article is to exhibit some families $\mathcal{F}_{n}$ of polyhedra with $n$ vertices on which there exists a point which admits $n$ antipodes, thereby proving that the upper bound given in [11] is the best one. Apart from an invocation of Theorem 0, due to A. D. Alexandrov, the construction will involve only very elementary mathematics.

In order to construct one of these polyhedra, we follow the modeler's way and begin to sketch its development on a proper cardboard (Section 2), and then, to cut it out, to fold it, and to glue it (Section 3). Section 4 is devoted to the proof that the obtained solid enjoys the desired property. Section 5 briefly discusses a way we can extend the described family. Section 6 illustrates and supplements the results of T. Zamfirescu. We prove that the family $\bigcup_{n} \mathcal{F}_{n}$ admits in its boundary some convex surface with quite a long set of antipodes, as well as surfaces on which some point admits a fractal set of antipodes. We also prove that the Hausdorff dimension of a set of antipodes on convex surfaces can take any real value between 0 and 1 .

As a matter of notation, the (non-oriented) angle with vertex $B$ and sides through $A$ and $C$ is denoted by $\widehat{A B C}$, as well as its measure. The parenthesis $(A B)$ stands for the line through $A$ and $B$, the bracket $[A B]$ for the line segment between $A$ and $B$. The (Euclidean) distance between $A$ and $B$ will be denoted by $A B$ or $\|A-B\|$. We reserve the notation $d(\mathcal{A}, \mathcal{B})$ for the (Euclidean) distance between sets, i.e. the $d(\mathcal{A}, \mathcal{B})=\inf _{A \in \mathcal{A}, B \in \mathcal{B}} A B$. The intrinsic distance between two points $a, b$ on a convex surface is denoted by $\rho(a, b)$. At last, the length of a rectifiable curve $\gamma$ is denoted by $L(\gamma)$. 


\section{Development}

Consider some unit circle $\mathcal{C}$ in the plane $\mathbb{R}^{2}$. Let $O$ be its center. Let $A, P$, $C_{N}, C_{N-1}, \ldots, C_{0}$, be $N+3$ points $(N \geq 1)$ lying in this order on one open half of $\mathcal{C}$, see Figure 1 . The development of our polyhedron will be entirely defined by the positions of these points, that is, by the $N+2$ positives numbers

$$
\begin{aligned}
\alpha & =\widehat{A O P} \\
\beta & =\widehat{P O C_{N}} \\
\gamma_{i} & =\widehat{C_{i} \widehat{O C_{i-1}}} \quad(i=1, \cdots, N)
\end{aligned}
$$

such that $\delta \stackrel{\text { def }}{=} \pi-\left(\alpha+\beta+\gamma_{1}+\ldots+\gamma_{N}\right)>0$. Let $\gamma$ be the sum of $\gamma_{i}$. The reflection with respect to $\left(O C_{0}\right)$ maps $C_{i}$ on $C_{i}^{\prime}(1 \leq i \leq N)$ and $P$ on $P^{\prime}$. The reflection with respect to $(O A)$ maps $P$ on $P^{\prime \prime}$. Let $C_{N}^{\prime \prime}$ be the second (i.e. distinct from $\left.C_{N}\right)$ point of intersection between $\left(C_{N} P^{\prime \prime}\right)$ and the circle of radius $A C_{N}$ centered at $A$. Let $B$ be the intersection point of the mediators of $\left[P^{\prime} P^{\prime \prime}\right]$ and $\left[C_{N}^{\prime} C_{N}^{\prime \prime}\right]$. We have the following result

Proposition 1. The polygon

$$
\mathfrak{P}_{1}=A P C_{N} C_{N-1} \ldots C_{1} C_{0} C_{1}^{\prime} \ldots C_{N}^{\prime} B C_{N}^{\prime \prime}
$$

together with the following set of rules $\mathfrak{R}_{1}$

- glue $\left(C_{0}, C_{1}\right)$ on $\left(C_{0}, C_{1}^{\prime}\right)$

- glue $\left(C_{i}, C_{i+1}\right)$ on $\left(C_{i}^{\prime}, C_{i+1}^{\prime}\right), 1 \leq i \leq N-1$

- glue $\left(A, C_{N}\right)$ on $\left(A, C_{N}^{\prime \prime}\right)$

- glue $\left(B, C_{N}^{\prime}\right)$ on $\left(B, C_{N}^{\prime \prime}\right)$

is the development of a non-degenerated polyhedron.

The proof of this result is postponed to Section 3, where the notion of development will be defined precisely. The polygon $\mathfrak{P}_{1}$ is shown in Figure 1. Before folding it into a polyhedron, we prove for further use the following three lemmas.

Lemma 1. Triangles $A P^{\prime \prime} C_{N}^{\prime \prime}$ and $A P C_{N}$ are congruent.

Proof. Obviously, we have

$$
A P=A P^{\prime \prime}, \quad A C_{N}=A C_{N}^{\prime \prime} .
$$

Since the triangle $A C_{N} C_{N}^{\prime \prime}$ is isosceles, we have

$$
\widehat{A C_{N}^{\prime \prime} P^{\prime \prime}}=\widehat{A C_{N} P^{\prime \prime}}=\frac{\alpha}{2}=\widehat{A C_{N} P} .
$$


Moreover

$$
\widehat{A P C_{N}}=\pi-\frac{\widehat{A O C_{N}}}{2}=\pi-\frac{\alpha+\beta}{2}
$$

and

$$
\widehat{A P^{\prime \prime} C_{N}^{\prime \prime}}=\pi-\widehat{A P^{\prime \prime} C_{N}}=\pi-\frac{\alpha+\beta}{2}=\widehat{A P C_{N}} .
$$

Hence the triangles are congruent.

Lemma 2. The point B lies inside the circle.

Proof. We will prove this by using complex numbers. In order to limit the amount of symbols, we identify the plane with $\mathbb{C}$ and use the same letter to designate both a point and its affix. We assume - without loss of generalitythat $\mathcal{C}$ is the unit circle and that $A=1$. It follows that

$$
P^{\prime \prime}=\frac{1}{P}, \quad C_{N}^{\prime \prime}=1+\frac{1-C_{N}}{P}, \quad C_{N}^{\prime}=\frac{C_{0}^{2}}{C_{N}}, \quad P^{\prime}=\frac{C_{0}^{2}}{P} .
$$

The point $B$ lies on the mediator of the segment $\left[P^{\prime} P^{\prime \prime}\right]$, whose equation is

$$
P\left(1-\frac{1}{C_{0}^{2}}\right) z+\frac{1}{P}\left(1-C_{0}^{2}\right) \bar{z}=0 .
$$

It also lies on the mediator of $\left[C_{N}^{\prime} C_{N}^{\prime \prime}\right]$, an equation of which is :

$$
\begin{aligned}
\left(\frac{C_{N}}{C_{0}^{2}}-1-P \frac{C_{N}-1}{C_{N}}\right) z+\left(\frac{C_{0}^{2}}{C_{N}}\right. & \left.-1-\frac{1-C_{N}}{P}\right) \bar{z} \\
& =1-\left(1+\frac{1-C_{N}}{P}\right)\left(1+P \frac{C_{N}-1}{C_{N}}\right) .
\end{aligned}
$$

Solving this linear system we get

$$
B=\frac{C_{0}^{2}\left(C_{N}-1\right)}{P\left(C_{N}-C_{0}^{2}\right)} .
$$

It follows that $B$ lies inside $\mathcal{C}$ if and only if $\left|C_{N}-A\right|<\left|C_{N}-C_{0}^{2}\right|$, which is obvious since the mediator of $\left[C_{0}^{2} A\right]$ is actually $\left(O C_{0}\right)$.

Lemma 3. The point $O$ lies inside the triangle $A C_{0} B$.

Proof. We prove the lemma by showing that $O$ can lie on none of the lines $(A B),\left(A C_{0}\right)$ or $\left(B C_{0}\right)$. So, the point $O$ has to be either always inside or always outside the triangle. It is easy to see, for some simple particular values (e.g. $\alpha=\beta=\gamma=\frac{\pi}{2}$ ), that $O$ actually lies inside. Since $\alpha+\beta+\gamma<\pi$, obviously $O \notin\left(A C_{0}\right)$. Assume that $O \in(A B)$. Since $B$ belongs to the mediator of $\left[P^{\prime} P^{\prime \prime}\right]$ which cuts $(O A)$ at $O$, necessarily $B=0$, in contradiction with (1). Assume now that $O \in\left(B C_{0}\right)$, i.e.

$$
\frac{B}{C_{0}}=\frac{C_{0}\left(C_{N}-1\right)}{P\left(C_{N}-C_{0}^{2}\right)} \in \mathbb{R}
$$




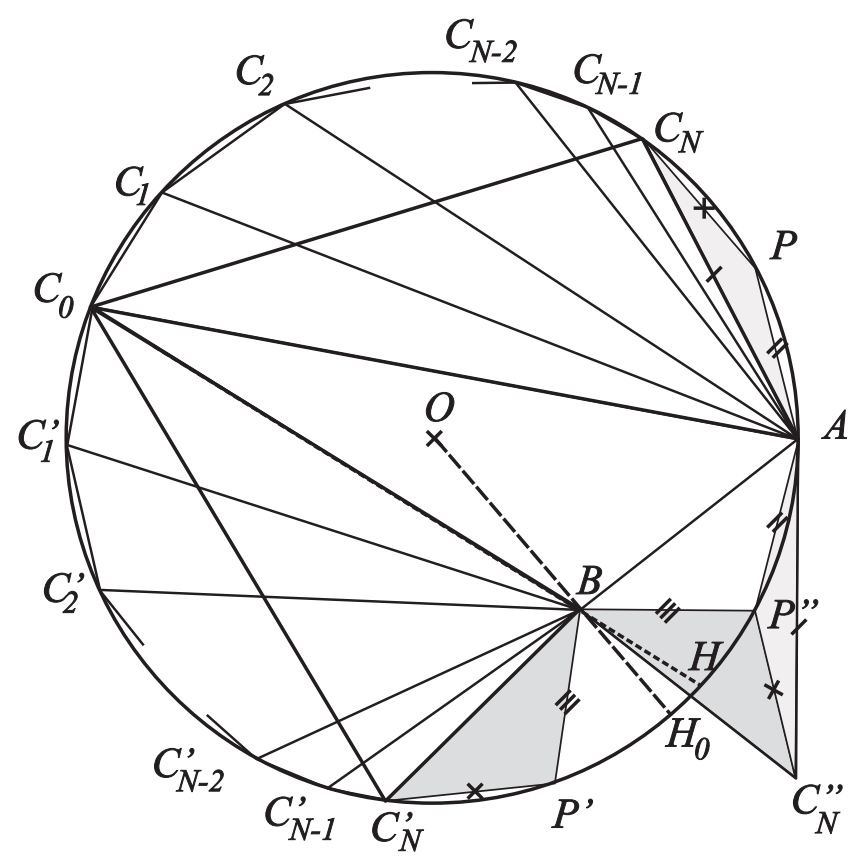

Figure 1: The development of the polyhedron.

A simple calculation shows that

$$
\frac{B}{C_{0}}-\overline{\left(\frac{B}{C_{0}}\right)}=\frac{C_{0}\left(C_{N}-1\right)\left(1-P^{2}\right)}{P\left(C_{N}-C_{0}^{2}\right)} \neq 0,
$$

and another contradiction is found.

\section{From development to polyhedron}

The fact that the above development will fold into a convex polyhedron follows from the (polyhedral version) of Alexandrov's gluing theorem [3]. In order to state it, we need to precise some points of vocabulary. A development $(\mathfrak{P}, \mathfrak{R})$ will be the data of one or several (planar) disjoint polygons, the union of which is denoted by $\mathfrak{P}$, together with a set $\mathfrak{R}$ of gluing rules. A gluing rule says which edge should be glued onto which other, in which direction. Hence a gluing rule is given by a pair of ordered pairs of vertices of the polygons. Of course, gluing rules cannot be chosen freely and have to satisfy some axioms, namely

- Each ordered pair involved in a gluing rule is the pair of endpoints of some edge of $\mathfrak{P}$. 
- Two edges glued together must have the same length.

- Each edge is glued to exactly one other edge.

It is clear that the polygon $\mathfrak{P}_{1}$ of the preceding section together with the set $\mathfrak{R}_{1}$ of gluing rules satisfies these axioms, so it is a development.

To any development, we can associate a metric space in the following way. The set of rules $\mathfrak{R}$ induces an equivalence relation, also denoted by $\mathfrak{R}$, in a natural way: $\mathfrak{R}$ is the smallest relation of equivalence such that, for any gluing rule of the form "glue $(X, Y)$ on $\left(X^{\prime}, Y^{\prime}\right)$ " and any $\lambda \in[0,1]$ the points $\lambda X+$ $(1-\lambda) Y$ and $\lambda X^{\prime}+(1-\lambda) Y^{\prime}$ are equivalent.

Denote by $\Pi$ the canonical surjection from $\mathfrak{P}$ to $\mathfrak{P} / \mathfrak{R}$. It is clear that a point which is not a vertex is equivalent to exactly one other point if it lies on an edge of the development, and to no other point if it belongs to the interior of $\mathfrak{P}$. A vertex is equivalent to an arbitrary number of points, which are clearly vertices too. A vertex of $\mathfrak{P} / \mathfrak{R}$ is by definition the equivalence class of some vertex of $\mathfrak{P}$. The curvature $\omega_{x}$ of some vertex $x=\left\{X_{1}, \ldots, X_{k}\right\} \in \mathfrak{P} / \mathfrak{R}$ is defined as $2 \pi$ minus the sum of the angles of $\mathfrak{P}$, measured toward $\mathfrak{P}$, at points $X_{i}$. If all but a finite number of points of some curve in the topological quotient $\mathfrak{P} / \mathfrak{R}$ lie in the image under $\Pi$ of the interior of $\mathfrak{P}$, then its (possibly infinite) length is well defined in a natural way. The distance between two points $x, y \in \mathfrak{P} / \mathfrak{R}$ will be by definition the infimum of the set of the lengths of those curves joining $x$ to $y$.

A development $\left(\mathfrak{P}^{\prime}, \mathfrak{R}^{\prime}\right)$ is said to be obtained from a development $(\mathfrak{P}, \mathfrak{R})$ by cutting and gluing, if one can 'cut' $\mathfrak{P}$ into finitely many polygons and move (apply an affine isometry of the plane) the obtained tiles in order to rebuild $\mathfrak{P}^{\prime}$, such that any two coinciding edges of two moved tiles were glued by some rule in $\mathfrak{R}$. Of course $\mathfrak{R}^{\prime}$ is derived from $\mathfrak{R}$ and the partitions of $\mathfrak{P}$ and $\mathfrak{P}^{\prime}$ in a natural way, namely the rules of $\mathfrak{R}^{\prime}$ are the rules of $\mathfrak{R}$, save those which correspond to some coinciding edges of moved tiles, together with the new rules that identify edges of the tiles which have been separated by cutting and moving.

Remark 1. If $\left(\mathfrak{P}^{\prime}, \mathfrak{R}^{\prime}\right)$ is obtained from $(\mathfrak{P}, \mathfrak{R})$ by cutting and gluing, then the quotients $\mathfrak{P}^{\prime} / \mathfrak{R}^{\prime}$ and $\mathfrak{P} / \mathfrak{R}$ are isometric $[3$, p. 51].

Consider the disjoint union of two isometric convex polygons $X_{0} X_{2} \ldots X_{k}$ and $Y_{0} Y_{2} \ldots Y_{k}$, labeled in such a way that the isometry maps $X_{i}$ on $Y_{i}$, and the $k+1$ gluing rules "glue $\left(X_{i}, X_{i+1}\right)$ on $\left(Y_{i}, Y_{i+1}\right)$ " (the index $i$ is taken modulo $k+1)$. The resulting metric space is called the double of $X_{0} X_{2} \ldots X_{k}$. It is not isometric to any convex surface. However it is easy to see that it is the limit of a sequence of prisms with fixed base $X_{0} X_{2} \ldots X_{k}$ and heights tending to zero. Such a metric space is called a degenerated convex polyhedron. Now we are in a position to state the polyhedral version of Alexandrov's gluing theorem [3].

Theorem 0. Let $(\mathfrak{P}, \mathfrak{R})$ be a development such that

1. $\mathfrak{P} / \mathfrak{R}$ is homeomorphic to the 2-dimensional sphere.

2. Each vertex of $\mathfrak{P} / \mathfrak{R}$ has non-negative curvature. 
Then, $\mathfrak{P} / \mathfrak{R}$ is isometric to the surface of a (possibly degenerated) convex polyhedron. Moreover the polyhedron is unique up to isometry.

Note that, with the above meaning of vertices, some vertices of $\mathfrak{P} / \mathfrak{R}$ (precisely those having a zero curvature) are not vertices of the resulting polyhedron.

Now, let us return to the proof of Proposition 1. We denote with a lowercase letter the vertex of $\mathfrak{P}_{1} / \mathfrak{R}_{1}$ corresponding to the vertices of $\mathfrak{P}_{1}$ which are denoted by the same uppercase letter(s). So $c_{N}=\left\{C_{N}, C_{N}^{\prime}, C_{N}^{\prime \prime}\right\}, b=\{B\}$, $c_{i}=\left\{C_{i}, C_{i}^{\prime}\right\}, 1 \leq i<N$, etc. It is quite clear that $\mathfrak{P}_{1} / \mathfrak{R}_{1}$ is homeomorphic to the sphere: one can easily compute the Euler characteristic in order to obtain a numerical argument.

It is also clear that the curvatures at points $a, b$ and $c_{0}$ are positive, since these equivalence classes are singletons. Let us compute

$$
\begin{aligned}
\omega_{c_{N}} & =2 \pi-A \widehat{C_{N} C_{N}}-1-B \widehat{C_{N}^{\prime} C_{N-1}^{\prime}}-\widehat{A C_{N}^{\prime \prime} B} \\
& =2 \pi-A \widehat{C_{N} C_{N}}-1-B \widehat{C_{N}^{\prime} C_{N-1}^{\prime}}-\widehat{A C_{N}^{\prime \prime} P^{\prime \prime}}-\widehat{P^{\prime \prime} C_{N}^{\prime \prime} B} \\
& =2 \pi-A \widehat{C_{N} C_{N}}-1-B \widehat{C_{N}^{\prime} C_{N-1}^{\prime}}-\widehat{A C_{N} P}-\widehat{B C_{N}^{\prime} P^{\prime}} \\
& =2 \pi-P \widehat{C_{N} C_{N-1}}-P^{\prime} \widehat{C_{N}^{\prime} C_{N-1}^{\prime}} \\
& =\beta+\gamma_{N}>0
\end{aligned}
$$

and, for $i=1, \ldots, N-1$,

$$
\omega_{c_{i}}=2 \pi-2 C_{i-1}{\widehat{C} C_{i} C_{i+1}}=C_{i+1} \widehat{O C}_{i-1}=\gamma_{i+1}+\gamma_{i}>0 .
$$

Hence Theorem 0 applies, and $\mathfrak{P}_{1} / \mathfrak{R}_{1}$ is isometric to the surface of some possibly degenerated convex polyhedron $\mathcal{P}$. Moreover, the curvatures of $a, b$, $c_{0}, \ldots, c_{N}$ are positive, whence $\mathcal{P}$ has exactly $N+3$ vertices.

Assume now that $\mathcal{P}$ is degenerated. So is it of the tetrahedron $a b c_{0} c_{N}$, which is obviously included (in the sense of inclusion of subsets of the 3-dimensional Euclidean space) in $\mathcal{P}$. In particular, the solid angle at vertex $c_{0}$ should vanish, that is, one of the following statements holds:

$$
\begin{aligned}
& \widehat{C_{N} C_{0} A}+\widehat{B C_{0} C_{N}^{\prime}}=\widehat{A C_{0} B} \\
& \widehat{B C_{0} C_{N}^{\prime}}+\widehat{A C_{0} B}=\widehat{C_{N} C_{0} A} \\
& \widehat{C_{N} C_{0} A}+\widehat{A C_{0} B}=\widehat{B C_{0} C_{N}^{\prime}} .
\end{aligned}
$$

Let $H$ be the other point of intersection of $\left(C_{0} B\right)$ and $\mathcal{C}$, and $H_{0}$ the midpoint of the circle-arc $P^{\prime \prime} P^{\prime}$. Since $O$ lies inside the triangle $A B C_{0}$ (Lemma 3), $H$ lies on the circle arc $H_{0} A$, whence

$$
\zeta \stackrel{\text { def }}{=} \widehat{A O H}<\alpha+\delta
$$

Note that

$$
\widehat{C_{N} C_{0} A}=\frac{\alpha+\beta}{2}, \quad \widehat{B C_{0} C_{N}^{\prime}}=\frac{2 \pi-2 \gamma-\alpha-\beta-\zeta}{2}, \quad \widehat{A C_{0} B}=\frac{\zeta}{2} .
$$


It follows that

$$
\widehat{C_{N} C_{0}} A+\widehat{B C_{0} C_{N}^{\prime}}-\widehat{A C_{0} B}=\pi-\gamma-\zeta>\beta>0
$$

whence (2) never holds. If (3) held, then $\left(A C_{0}\right)$ would be the bisector of $\widehat{C_{N} C_{0} C_{n}^{\prime}}$, i.e. $\left(A C_{0}\right)=\left(O C_{0}\right)$, which is obviously impossible. In the same way, (4) would infer $O \in\left(B C_{0}\right)$, which is impossible by virtue of Lemma 3 . It follows that the polyhedron $\mathcal{P}$ is not degenerated. This ends the proof of Proposition 1.

\section{As many antipodes as vertices}

The main result is now almost at hand. It remains to prove that $a, p, c_{0}, \ldots, c_{N}$ are actually antipodes of $o$. We need for this purpose the following

Lemma 4. [6, (13.10) and (13.11)] On a convex surface, if two shortest paths have a common segment, then one of is incuded in the other. It follows that, if $\sigma$ is a shortest path from $x$ to $y$, and $z$ is a point interior to $\sigma$, then the portion of $\sigma$ delimitated by $x$ and $z$ is the only shortest path from $x$ to $z$.

Remark 2. The above lemma can be stated in a quite more general frame. It is indeed a basic property of Alexandrov spaces with curvature bounded below (see for instance [5] or [12]).

Theorem 1. The point $o \in \mathcal{P}$ has exactly $N+3$ antipodes, namely $a, c_{0}, \ldots, c_{N}$, and $p$.

Proof. Our first claim is that the distance between $o$ and any point of $\mathcal{P}$ is never greater than 1 . Consider the polygon

$$
\mathfrak{P}_{2}=C_{0} C_{1} \ldots C_{N} P A P^{\prime \prime} B P^{\prime} C_{N}^{\prime} C_{N-1}^{\prime} \ldots C_{2}^{\prime} C_{1}^{\prime}
$$

and the following set $\mathfrak{R}_{2}$ of gluing rules

- Glue $\left(C_{0}, C_{1}\right)$ on $\left(C_{0}, C_{1}^{\prime}\right)$

- Glue $\left(C_{i}, C_{i+1}\right)$ on $\left(C_{i}^{\prime}, C_{i+1}^{\prime}\right), 1 \leq i<N$

- Glue $(A, P)$ on $\left(A, P^{\prime \prime}\right)$

- Glue $\left(B, P^{\prime \prime}\right)$ on $\left(B, P^{\prime}\right)$

- Glue $\left(P^{\prime}, C_{N}^{\prime}\right)$ on $\left(P, C_{N}\right)$.

Since the triangles $A P C_{N}$ and $A P^{\prime \prime} C_{N}^{\prime \prime}$, and the triangles $B P^{\prime \prime} C_{N}^{\prime \prime}$ and $B P^{\prime} C_{N}^{\prime}$ are congruent, $\left(\mathfrak{P}_{2}, \mathfrak{R}_{2}\right)$ is obtained from $\left(\mathfrak{P}_{1}, \mathfrak{R}_{1}\right)$ by cutting (along $\left.\left[P^{\prime \prime} A\right] \cup\left[P^{\prime \prime} B\right] \cup\left[P^{\prime \prime} C^{\prime \prime}\right]\right)$ and gluing $\left(\left(C_{N}^{\prime \prime}, A\right)\right.$ on $\left(C_{N}, A\right)$ and $\left(C_{N}^{\prime \prime}, B\right)$ on $\left.\left(C_{N}^{\prime}, B\right)\right)$, whence they are developments of the same polyhedron $\mathcal{P}$ (see Remark 1). Since $\mathfrak{P}_{2}$ is included in the unit disc, the distance from $o$ cannot exceed 1. 
Our second claim is that any shortest path $\sigma$ between $o$ and any other point of $\mathcal{P}$ corresponds to a line segment of $\mathfrak{P}_{2}$. In other words, such a path cannot cross

$$
\Gamma \stackrel{\text { def }}{=}[a p] \cup[b p] \cup\left[c_{n} p\right] \cup \bigcup_{i=0}^{N-1}\left[c_{i} c_{i+1}\right] .
$$

Assume on the contrary that $\sigma$ is crossing $\Gamma$ and denote by $y \in \mathcal{P}=\mathfrak{P}_{2} / \mathfrak{R}_{2}$ the first (counted from $o$ ) crossing point. As a minimizing geodesic, $\sigma$ cannot pass through any vertex (see for instance $[6,(12.4)]$ ), so $y$ should lie in the relative interior of one of the segments $[a p],[b p],\left[c_{n} p\right],\left[c_{i} c_{i+1}\right]$. Hence $y$ is a pair $\left\{Y, Y^{\prime}\right\}$ of points of $\mathfrak{P}_{2}$, and the part of $\sigma$ between $o$ and $y$ is unfolded into some line segment $[O Y]$ or $\left[O Y^{\prime}\right]$, say $[O Y]$. Note that $Y^{\prime}$ is the point symmetric to $Y$ with respect to $(O A)$ (if $y \in[a p]$ ), to $\left(O H_{0}\right)$ (if $y \in[b p]$ ) or to $\left(O C_{0}\right)$ (in the other cases), whence $O Y=O Y^{\prime}$. The path of $\mathcal{P}$ corresponding to $\left[O Y^{\prime}\right]$ is also a shortest path between $o$ and $y$, in contradiction with Lemma 4. This prove our second claim.

It follows that, for any $x \in \mathcal{P}=\mathfrak{P}_{2} / \mathfrak{R}_{2}$ and any $X \in x, \rho(o, x)$ equals $O X$, whence $\rho(o, p)=\rho\left(o, c_{i}\right)=\rho(o, a)=1$.

Remark 3. The curve $\Gamma$ is the cut-locus of $o$.

In order to conclude the section, we will examine what happens when one or several parameters $\alpha, \beta, \gamma_{1}, \ldots, \gamma_{N}, \delta$ vanish.

First, there is almost nothing to say when one or several (but not all) $\gamma_{i}$ vanish: this case reduce to a lower $N$ case. If all $\gamma_{i}$ vanish, then $B \in \mathcal{C}$ and the resulting $\mathcal{P}$ is nothing but the double of the acute triangle $A B C_{0}$. The point $p$ is the orthocentre of the face $a b c_{0}$ which does not contain $o$. The point $o$ has 4 antipodes $\left(a, b, c_{0}\right.$, and $\left.p\right)$, while $\mathcal{P}$ has only three vertices. However, since $\mathcal{P}$ is degenerated, this is not in contradiction with the main result of [11]. If $\beta=0$, then $p=c_{N}$. The $(N+3)$-vertex polyhedron does not degenerate, but $o$ has only $N+2$ antipodes. If $\alpha=0$ (i.e. $\left.A=P=P^{\prime \prime}\right)$ then $O$ belongs to $\left(C_{0} B\right)$ and $\mathcal{P}$ is the double of the $(N+3)$-gon $A B C_{0} \ldots C_{N}$. At last if $\delta=0$, then $B=P^{\prime}=P^{\prime \prime}, O$ lies on $\left(O C_{0}\right)$ and $\mathcal{P}$ is the double of $A C_{0} \ldots C_{N} P$.

\section{A larger family of tetrahedra}

In the above examples, all antipodes of $o$ but one are vertices. Moreover, the only non-vertex antipode of $o$ is joined to $o$ by three distinct shortest paths. Whether these properties are enjoyed by all polyhedra with $n$ vertices admitting a point with $n$ antipodes is a natural question. Some earlier works give a partial answer. The fact that a non-vertex antipode of any point on any polyhedron is always joined by at least three minimizing geodesics is indeed one of the most basic results on this subject [10]. It is also proved in [11] that for such a polyhedron, at least two of the antipodes of the distinguished point should be very acute (i.e. with curvature more than $\pi$ ) vertices. This section is devoted to showing that, in the case of the tetrahedron, the two other points can be non-vertex points. 


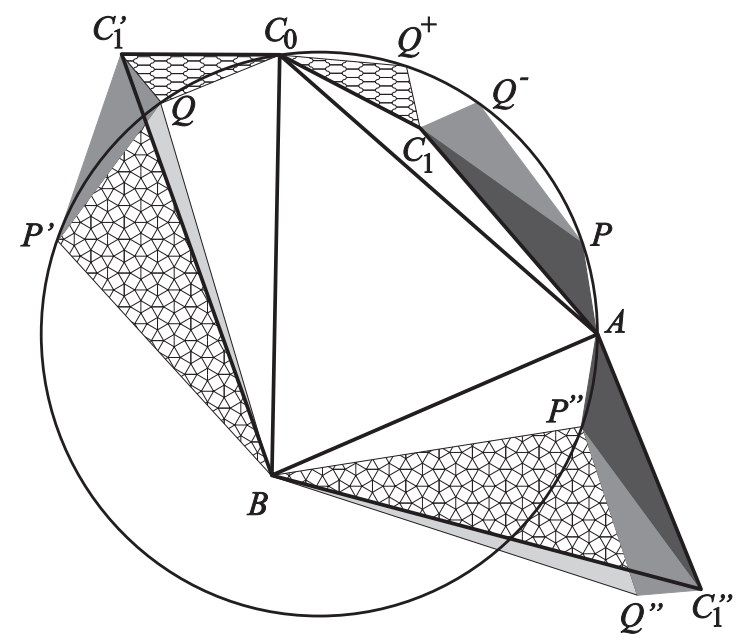

Figure 2: The development of the tetrahedron. Triangles filled with the same gray or pattern are isometric.

In this section, we assume $N=1$. The main idea is to give a deformation $\mathcal{P}(\varepsilon)$ of the preceding example $\mathcal{P}=\mathcal{P}(0)$ in such a way that $c_{1}$ is no more an antipode (i.e. $C_{1}$ lies inside the circle). As explained before, the point $q$ which will become an antipode of $o$ instead of $c_{1}$ must be joined to $o$ by (at least) three shortest paths. As, on $\mathcal{P}(0)$, there were only two shortest paths between $o$ and $c_{1}$, one of them has to split. Consider a point $q \in \mathcal{P}(\varepsilon)$ somewhere beyond $c_{1}$, looking from $o$. If $q$ is near enough to $c_{1}$ then a shortest path $\sigma$ between $o$ and $q$ should lie in a neighborhood of the only shortest path between $o$ and $c_{1}$. By moving $q$ on a right-left (still from $o$ point of view) degree of liberty, we can ensure that $\sigma$ lies either on the right side, or on the left side of $c_{1}$. Since a shortest path never passes through a vertex $[6,(12.4)]$, there is a right-left position such that two shortest paths, one on each side, exist. Now, if the tetrahedron is not too deformed, by moving $q$ backward, we will obtain a third shortest path, close to the second one between $o$ and $c_{1}$ on $\mathcal{P}(0)$. It is not clear, that the deformation could be done in a way that satisfies the equation $\rho(o, q)=1$. However the space of tetrahedra, up to affine isometries is 6-dimensional, and the fact that $A, C_{0}, P, P^{\prime}$ and $P^{\prime \prime}$ all lie on a unit circle is described by three equations. Hence it is natural to conjecture that $\mathcal{P}$ can be deformed without breaking the fact that $a, p, c_{0}$ are antipodes of $o$ with three degrees of liberty. If we ask moreover that the deformation satisfies $\rho(o, q)=1$, there still remain two degrees of liberty. One of them corresponds to the variation of $\gamma$; it remains one parameter $\varepsilon$. This heuristic description is far beyond a proof, hence we will present a more formal construction.

Choose three positive numbers $\alpha, \beta, \gamma$ such that $\alpha+\beta+\gamma<\pi$. For $\varepsilon>0$ 
small enough, consider the points

$$
\begin{aligned}
A & =(1,0) \\
C_{0} & =(\cos (\alpha+\beta+\gamma), \sin (\alpha+\beta+\gamma)) \\
P & =(\cos \alpha, \sin \alpha) \\
Q^{+} & =(\cos (\alpha+\beta+\varepsilon), \sin (\alpha+\beta+\varepsilon)) \\
Q^{-} & =(\cos (\alpha+\beta-\varepsilon), \sin (\alpha+\beta-\varepsilon)),
\end{aligned}
$$

all lying on the unit circle $\mathcal{C}$. Let $Q$ be the point symmetric to $Q^{+}$with respect to $\left(O C_{0}\right)$. Let $P^{\prime}$ be the point of $\mathcal{C}$ such that $Q P^{\prime}=Q^{-} P$ and $C_{0}, Q, P^{\prime}$ lie on $\mathcal{C}$ in the direct order. Let $R_{1}$ be the rotation which maps the ordered pair $\left(Q^{-}, P\right)$ on $\left(Q, P^{\prime}\right)$. Let $R_{2}$ be the rotation of center $C_{0}$ which maps $Q^{+}$on $Q$. The images under $R_{1}$ and $R_{2}$ of the mediator of $\left[Q^{+} Q^{-}\right]$intersect at $C_{1}^{\prime}$; put $C_{1}=R_{2}^{-1}\left(C_{1}^{\prime}\right)$. Let $P^{\prime \prime}$ be the point symmetric to $P$ with respect to $(O A)$. The rotation of center $A$ which maps $P$ on $P^{\prime \prime}$ maps $C_{1}$ on $C_{1}^{\prime \prime}$. The mediators of $\left[P^{\prime} P^{\prime \prime}\right]$ and $\left[C_{1}^{\prime} C_{1}^{\prime \prime}\right]$ intersect at $B$.

Figure 2 illustrates the above construction. We let the reader check that the polygon

$$
\mathfrak{P}_{1}=A C_{1}^{\prime \prime} B C_{1}^{\prime} C_{0} C_{1}
$$

together with the following set of rules $\Re_{1}$

- glue $\left(C_{0}, C_{1}\right)$ on $\left(C_{0}, C_{1}^{\prime}\right)$

- glue $\left(A, C_{1}\right)$ on $\left(A, C_{1}^{\prime \prime}\right)$

- glue $\left(B, C_{1}^{\prime}\right)$ on $\left(B, C_{1}^{\prime \prime}\right)$

is (for small $\varepsilon$ ) the development of a tetrahedron with vertices $a, b, c_{0}, c_{1}$. For $\varepsilon=0$, we get the tetrahedron described in the preceding sections.

Consider the polygon

$$
\mathfrak{P}_{2}=A C_{1}^{\prime \prime} Q^{\prime \prime} B Q C_{1}^{\prime} C_{0} C_{1}
$$

together with the following set of rules $\mathfrak{R}_{2}$

- glue $\left(C_{0}, C_{1}\right)$ on $\left(C_{0}, C_{1}^{\prime}\right)$

- glue $\left(A, C_{1}\right)$ on $\left(A, C_{1}^{\prime \prime}\right)$

- glue $\left(Q, C_{1}^{\prime}\right)$ on $\left(Q^{\prime \prime}, C_{1}^{\prime \prime}\right)$

- glue $(B, Q)$ on $\left(B, Q^{\prime \prime}\right)$.

Since the triangles $B Q C_{1}^{\prime}$ and $B Q^{\prime \prime} C_{1}^{\prime \prime}$ are congruent, it is clear that $\left(\mathfrak{P}_{2}, \mathfrak{R}_{2}\right)$ is obtained from $\left(\mathfrak{P}_{1}, \mathfrak{R}_{1}\right)$ by cutting and gluing and so, is development of the same tetrahedron (see Remark 1).

Consider now the polygon

$$
\mathfrak{P}_{3}=A P^{\prime \prime} B P^{\prime} Q C_{0} Q^{+} C_{1} Q^{-} P
$$

together with the following set of rules $\mathfrak{R}_{3}$ 


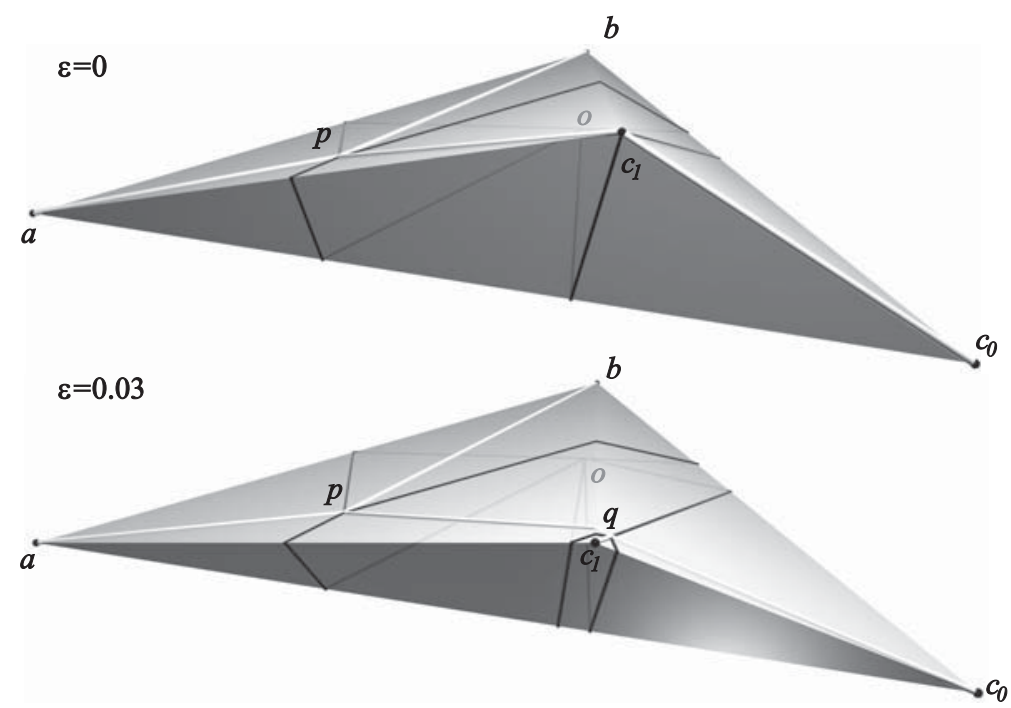

Figure 3: The tetrahedra $\mathcal{P}(0)$ and $\mathcal{P}(0.03)$.

- glue $\left(C_{0}, Q\right)$ on $\left(C_{0}, Q^{+}\right)$

- glue $\left(C_{1}, Q^{+}\right)$on $\left(C_{1}, Q^{-}\right)$

- glue $\left(Q, P^{\prime}\right)$ on $\left(Q^{-}, P\right)$

- glue $(A, P)$ on $\left(A, P^{\prime \prime}\right)$

- glue $\left(B, P^{\prime}\right)$ on $\left(B, P^{\prime \prime}\right)$.

Since the triangles $B Q P^{\prime}$ and $B Q^{\prime \prime} P^{\prime \prime}$, the triangles $C_{0} C_{1}^{\prime} Q$ and $C_{0} C_{1} Q^{+}$, the triangles $C_{1} P Q^{-}$and $C_{1}^{\prime \prime} P^{\prime \prime} Q^{\prime \prime}$ and the triangles $C_{1} A P$ and $C_{1}^{\prime \prime} A P^{\prime \prime}$ are congruent, it is clear that $\left(\mathfrak{P}_{3}, \mathfrak{R}_{3}\right)$ is obtained from $\left(\mathfrak{P}_{2}, \mathfrak{R}_{2}\right)$ by cutting and gluing, and so, is a development of the same tetrahedron (see Remark 1). Since $\mathfrak{P}_{3}$ is included in the unit disc, the distance from $o$ cannot exceed 1 . Thus, the antipodes of $o$ are $a, c_{0}, p$ and $q$. The non-vertex antipodes of $o$ are both joined to $o$ by three shortest paths.

Figure 3 represents $\mathcal{P}(0)$ and $\mathcal{P}(0.03)$ for $\alpha=\beta=\gamma=\frac{\pi}{6}$. The white thick line is the set of those points which are joined to $o$ by at least two shortest paths, and the black lines are the shortest paths from $o$ to $p$ and from $o$ to $q$.

\section{$6 \quad$ Fractal sets of antipodes}

The section is devoted to the study of limit cases, when $N$ goes to infinity. The goal of the section is to prove the following 
Theorem 2. Let $\mathcal{H}$ be an open half-circle. Let $K$ be a compact subset of $\mathcal{H}$ and $U$ a point which does not belong to $K$. Then there exists a convex surface $\mathcal{S}$ and a point $o \in \mathcal{S}$ such that the set of antipodes of o in $\mathcal{S}$, endowed with the intrinsic distance of $\mathcal{S}$, is locally isometric to $K \cup\{U\}$, endowed with the Euclidean distance of $\mathbb{R}^{2}$.

The main idea is easy: given a compact subset $K$ of a half circle, we can choose a sequence of finite sets approaching it. For each finite set $F$, the preceding section provides a polyhedron $\mathcal{P}_{F}$ admitting $F$ as a set of antipodes, and $\mathcal{S}$ will be the limit of these polyhedra. However, comparatively to the preceding sections, this proof is somewhat long and technical, and requires not less than ten lemmas.

First, we recall the elementary tools we will have to use. The Hausdorff distance between two compact subsets $K_{1}, K_{2} \subset \mathbb{R}^{d}$ is the smallest number $r$ such that each point of $K_{1}$ is included in a closed ball of radius $r$ centered at some point of $K_{2}$, and conversely. The Hausdorff distance is actually a distance on the set of non-empty compact subsets of $\mathbb{R}^{d}$. We denote it by $h(\cdot, \cdot)$. From now on, convergence of a sequence of compacts is understood with respect to the Hausdorff distance. We will use some well-known facts about this distance, namely that each bounded sequence of compacts in $\mathbb{R}^{d}$ admits a converging subsequence, that the finite subsets are dense in the set of all non-empty compact subsets, and that the set of convex compact subsets is closed in the space of all nonempty compact subsets. The latter fact admits as a corollary that the limit of a converging sequence of convex surfaces (embedded in $\mathbb{R}^{3}$ ) is either a convex surface, or a convex body of dimension less than 3 . The following lemma [6, p. 81] states that, if the limit is a convex surface, then the intrinsic distances also converge.

Lemma 5. Let $\mathcal{S}_{n}$ be a sequence of convex surfaces converging to a convex surface $\mathcal{S}$. Denote by $\rho_{n}$ (respectively $\rho$ ) the intrinsic distance on $\mathcal{S}_{n}$ (respectively $\mathcal{S})$. Let $x_{n}, y_{n}$ be points of $\mathcal{S}_{n}$ such that the sequences $\left(x_{n}\right)_{n}$ and $\left(y_{n}\right)_{n}$ are converging respectively to $x$ and $y$ in $\mathbb{R}^{3}$. Then $x, y \in \mathcal{S}$ and $\rho_{n}\left(x_{n}, y_{n}\right)$ tends to $\rho(x, y)$.

Concerning the notions of Hausdorff measure and Hausdorff dimension, we refer (for instance) to [4]. We recall that the $d$-dimensional Hausdorff measure is preserved by isometries, and that the 1-dimensional measure of a simple curve coincides with its length. We also recall that the Hausdorff dimension is preserved by any bi-Lipschitz map. Since the intrinsic distance of a convex surface and the restriction of the Euclidean distance of $\mathbb{R}^{3}$ are equivalent (see $[6$, p. 78]), the Hausdorff dimension of a subset of convex surface does not depend on the considered distance.

Denote by $\mathcal{C}_{u}^{v}$ the circle $\operatorname{arc}\{(\cos t, \sin t) \mid u<l<v\}$. We fix two positive numbers $\alpha, \delta$ such that $\alpha+\delta<\pi$. To any finite subset $F$ of $\mathcal{C}_{\alpha}^{\pi-\delta}$, we associate the development $\left(\mathfrak{P}_{F}, \mathfrak{R}_{F}\right)$, corresponding to the development $\left(\mathfrak{P}_{2}, \mathfrak{R}_{2}\right)$ of Section 4, where $N=\operatorname{card}(F), A=(1,0), P=(\cos \alpha, \sin \alpha), C_{0}=(-\cos \delta, \sin \delta)$ 


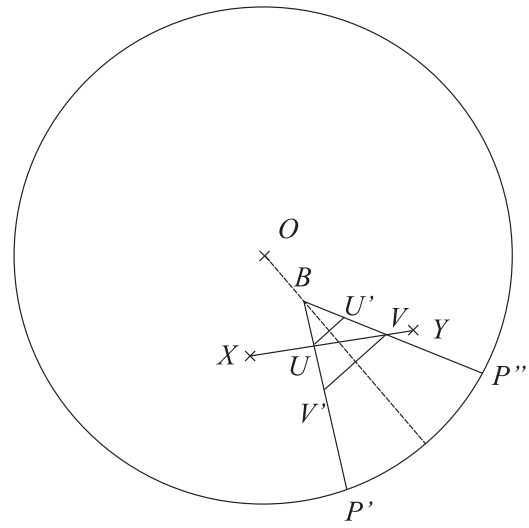

Figure 4: Definition of $U, V, U^{\prime}$ and $V^{\prime}$ in Lemma 6.

and $C_{1}, \ldots, C_{N}$ are the points of $F$, numbered from left to right. We put $\beta \stackrel{\text { def }}{=} \widehat{P O C_{N}}$ and $\gamma \stackrel{\text { def }}{=} \widehat{C_{N} O C_{0}}$. The development $\left(\mathfrak{P}_{F}, \mathfrak{R}_{F}\right)$ folds into some polyhedron $\mathcal{P}_{F}$, uniquely defined up to isometry. If we assume moreover that $o=(0,0,0), a=(1,0,0), b=\left(x_{b}, y_{b}, 0\right)$ with $y_{b}>0$ and that the third coordinate of $c_{N}$ is positive, then $\mathcal{P}_{F}$ is uniquely defined as a subset of $\mathbb{R}^{3}$. This allows us to define the folding map $\Phi_{F}: \mathfrak{P}_{F} \rightarrow \mathbb{R}^{3}$. For further use we prove the

Lemma 6. Let $X, Y$ be two points of $\mathfrak{P}_{F}$. The distance on $\mathcal{P}_{F}$ between $\Phi_{F}(X)$ and $\Phi_{F}(Y)$ is not greater than $X Y$.

Proof. If the segment $[X Y]$ lies in $\mathfrak{P}_{F}$, then $\Phi_{F}([X Y])$ is a path between $\Phi_{F}(X)$ and $\Phi_{F}(Y)$, and the result holds. Assume now that $[X Y]$ is crossing the segments $\left[B P^{\prime}\right]$ and $\left[B P^{\prime \prime}\right]$. Denote by $U, V, U^{\prime}$ and $V^{\prime}$ the points of intersection and their images under the reflection with respect to $(O B)$, labeled as on Figure 4. We claim that $\Phi_{F}([X U]) \cup \Phi_{F}\left(\left[U V^{\prime}\right]\right) \cup \Phi_{F}([V Y])$ is a path shorter than $[X Y]$. Indeed, it is sufficient to prove that $U V>U V^{\prime}$. This is so because in the triangle $U V V^{\prime}$,

$$
\widehat{U V^{\prime} V}=\widehat{U^{\prime} V V^{\prime}}>\widehat{U V V^{\prime}} .
$$

Lemma 7. Let $\gamma:\left[t_{0}, t_{1}\right] \rightarrow \mathbb{R}^{2}$ be a simple parametrization of a polygonal line inscribed in a half circle. Let $\tau \in\left[t_{0}, t_{1}\left[\right.\right.$. The function $f:\left[\tau, t_{1}\right] \rightarrow \mathbb{R}$ defined by $f(t)=\|\gamma(t)-\gamma(\tau)\|$ is increasing.

Proof. To prove the result, it is sufficient to notice that, on the one hand, the restriction of $f$ to any subinterval of $\left[t_{0}, t_{1}\right]$ whose image is a line segment is monotone, and on the other hand, its restriction to the inverse image of the set of vertices is increasing. The details are left to the reader. 
Lemma 8. Let $\mathcal{L}=\left[P C_{N}\right] \cup \bigcup_{k=1}^{N}\left[C_{k-1} C_{k}\right] \subset \mathfrak{P}_{F}$. There exists a positive number $r$ depending only on $\alpha, \delta$ such that, for all $X \in \mathcal{L}$, the restriction of $\Phi_{F}$ to the intersection of $\mathcal{L}$ with a ball of radius $r$ centered at $X$ is an isometry with respect to the intrinsic distance of $\mathcal{P}_{F}$.

Proof. We denote by $S$ the symmetry with respect to $\left(O C_{0}\right)$. Let $\mathcal{H}_{1}$ be the convex hull of $\{O\} \cup \mathcal{C}_{-\alpha-\delta}^{0}, \mathcal{H}_{2}$ the one of $\mathcal{C}_{\alpha}^{\pi-\delta}$ and $\mathcal{H}_{3}$ the one of $\mathcal{C}_{\pi-\delta}^{2 \pi-2 \delta-\alpha}$. Let $r=d\left(\mathcal{H}_{1}, \mathcal{H}_{2} \cup \mathcal{H}_{3}\right) ; r$ is clearly not greater than the distance between a point of $\left[A P^{\prime \prime}\right] \cup\left[P^{\prime \prime} B\right]$ and a point of $\mathcal{L} \cup S(\mathcal{L})$.

Assume that $X Y<r$ and that the abscissa of $X$ is less than the one of $Y$ (otherwise exchange them). Put $X^{\prime}=S(X), Y^{\prime}=S(Y), x=\Phi_{F}(X)$ and $y=\Phi_{F}(Y)$. Let $\sigma$ be a minimizing geodesic of $\mathcal{P}_{F}$ between $x$ and $y$. If $\sigma$ does not cross $\mathcal{M} \stackrel{\text { def }}{=} \Phi_{F}(\mathcal{L}) \cup[b p] \cup[a p]$, then there exist two points $X^{*} \in\left\{X, X^{\prime}\right\}$ and $Y^{*} \in\left\{Y, Y^{\prime}\right\}$ such that $\sigma=\Phi_{F}\left(\left[X^{*} Y^{*}\right]\right)$. Since $\Phi_{F}([X Y])$ is a path from $x$ to $y$ of length $X Y=X^{\prime} Y^{\prime}<X Y^{\prime}=X^{\prime} Y$ we have either $\left(X^{*}, Y^{*}\right)=(X, Y)$ or $\left(X^{*}, Y^{*}\right)=\left(X^{\prime}, Y^{\prime}\right)$, and so $\rho(x, y)=X Y$.

Assume now that $\sigma$ intersects $\mathcal{M}$ and let $u$ be the first (from $x$ ) point of intersection of $\sigma$ with $\mathcal{M}$. Let $\sigma_{u}$ be the part of $\sigma$ delimitated by $x$ and $u$. Obviously, $\sigma_{u}$ is the image under $\Phi_{F}$ of some segment $\left[X^{*} U^{*}\right]$, with $X^{*} \in$ $\left\{X, X^{\prime}\right\}$ and $\Phi_{F}\left(U^{*}\right)=u$. We discuss three cases.

Case 1: $u \in \Phi_{F}(\mathcal{L})$. In this case $U^{*} \in \mathcal{L} \cup S(\mathcal{L})$ and $\Phi_{F}\left(S\left(\left[X^{*} U^{*}\right]\right)\right)$ is another shortest path from $x$ to $u$, in contradiction with Lemma 4 .

Case 2: $u \in[a p]$. Let $U \in[A P]$ and $U^{\prime \prime} \in\left[A P^{\prime \prime}\right]$ be such that $\Phi_{F}^{-1}(u)=$ $\left\{U, U^{\prime \prime}\right\}$. Assume first that $X^{*}=X$. By Lemma 7 we have $X Y<X U$ and since

$$
\begin{aligned}
\rho(x, y) & \leq L\left(\Phi_{F}([X Y])\right)=X Y<X U \\
& =\min \left(X U, X U^{\prime \prime}\right)=L\left(\sigma_{u}\right)<\rho(x, y),
\end{aligned}
$$

we get a contradiction. Hence $X^{*}=X^{\prime}$, and, since $X U<X^{\prime} U, U^{*}=U^{\prime \prime}$. Now

$$
\rho(x, y) \leq X Y<r<X^{\prime} U^{\prime \prime}=L\left(\sigma_{u}\right)<\rho(x, y),
$$

and we get another contradiction.

Case 3: $u \in[b p]$. Let $U^{\prime} \in\left[B P^{\prime}\right]$ and $U^{\prime \prime} \in\left[B P^{\prime \prime}\right]$ be such that $\Phi_{F}^{-1}(u)=$ $\left\{U^{\prime}, U^{\prime \prime}\right\}$. Assuming that $U^{*}=U$ would infer

$$
\rho(x, y) \leq X Y<r \leq X U^{*}=L\left(\sigma_{u}\right)<\rho(x, y),
$$

whence $U^{*}=U^{\prime}$. Let $v$ be the next (along $\sigma$, from $x$ to $y$ ) point of $\sigma \cap \mathcal{M}$ and denote by $\sigma_{v}$ the part of $\sigma$ delimitated by $u$ and $v$. Let $V^{\prime \prime}$ be the point such that $\sigma_{v}=\Phi_{F}\left(\left[U^{\prime \prime} V^{\prime \prime}\right]\right)$. Since $U^{\prime \prime} V^{\prime \prime}=L\left(\sigma_{V}\right)<\rho(x, y) \leq X Y<r$, $V^{\prime \prime} \in\left[A P^{\prime \prime}\right]$. Let $V \in[A P]$ be the point symmetric to $V^{\prime \prime}$ with respect to $(O A)$. The next (along $\sigma$, from $x$ to $y$ ) point $w$ of $\sigma \cap \mathcal{M}$ obviously belongs to $\Phi_{F}(\mathcal{L})$. Let $\sigma_{w}$ be the part of $\sigma$ delimitated by $v$ and $w$, we have $\sigma_{w}=\Phi_{F}([V W])$ for some point $W \in \mathcal{L} \cup S(\mathcal{L})$. Moreover, since $S(\mathcal{L})$ is included in the half plane 
bounded by $\left(O C_{0}\right)$ not containing $V, W$ must lie on $\mathcal{L}$. We claim that $W=Y$. Assume on the contrary that $\sigma$ is crossing $\Phi_{F}(\mathcal{L})$ at point $w$ and denote by $z$ the next point of $\mathcal{M} \cap \sigma$. Since $\sigma$ cannot visit twice the face $a p c_{N}$ of $\mathcal{P}_{F}([1]), z$ belongs to $\Phi_{F}(\mathcal{L})$. Hence the part $\sigma_{z}$ of $\sigma$ delimitated by $w$ and $z$ is the image under $\Phi_{F}$ of some segment $[S(W) Z]$, for some point $Z \in \mathcal{L} \cup S(\mathcal{L})$. In this case $\Phi_{F}([W S(Z)])$ is also a shortest path from $w$ to $z$, in contradiction with Lemma 4. This proves the claim. Let $R_{1}$ be the rotation of center $B$ which maps $P^{\prime \prime}$ on $P^{\prime}, R_{2}$ the one of center $A$ which maps $P$ on $P^{\prime \prime}$, and put $R=R_{1} \circ R_{2}$. Clearly $L(\sigma)=X^{\prime} R(Y)$. Since $R(P)=P^{\prime}$, we have $R(Y) P^{\prime}=P Y=P^{\prime} Y^{\prime}$, whence $P^{\prime}$ belongs to the mediator of $\left[Y^{\prime} R(Y)\right]$. Similarly, $R$ maps $C_{N}$ on $C_{N}^{\prime}$, whence the mediator of $\left[R(Y) Y^{\prime}\right]$ is actually $\left(P^{\prime} C_{N}^{\prime}\right)$. It follows that $\rho(x, y) \leq X Y=X^{\prime} Y^{\prime} \leq X^{\prime} R(Y)=L(\sigma)=\rho(x, y)$. This completes the third case and the theorem.

Denote by $\tilde{\mathfrak{P}}$ the compact plane domain (depending on $\alpha, \delta$ ) delimitated by the line segments $[P A],\left[A P^{\prime \prime}\right],\left[P^{\prime \prime} H_{0}\right],\left[H_{0} P^{\prime}\right]$, and the circle $\operatorname{arc} \mathcal{C}_{\alpha}^{2 \pi-\alpha-2 \delta}$. It is clear that $\tilde{\mathfrak{P}}$ contains $\mathfrak{P}_{F}$. The complement of $\mathfrak{P}_{F}$ in $\tilde{\mathfrak{P}}$ has $2 N+3$ connected components. One of them, denoted by $\mathcal{Q}_{F}$ is delimitated by the quadrilateral $B P^{\prime \prime} H_{0} P^{\prime}$. All others components are delimitated by a circle arc and the corresponding chord. We denote the union of these components by $\mathcal{T}_{F}$. We define $\Pi_{F}: \tilde{\mathfrak{P}} \rightarrow \mathfrak{P}_{F}$ as follows: if $X \in \mathfrak{P}_{F}$, then $\Pi_{F}(X)=X$. If $X \in \mathcal{T}_{F}$ then $\Pi_{F}(X)$ is the orthogonal projection of $X$ onto the maximal segment included in the boundary of the connected component of $\mathcal{T}_{F}$ containing $X$. Finally, if $X$ belongs to $\mathcal{Q}_{F}$, then $\Pi_{F}(X)$ is the point of $\left[P^{\prime} B\right] \cup\left[B P^{\prime \prime}\right]$ such that the line $\left(X \Pi_{F}(X)\right)$ is parallel to $\left(O H_{0}\right)$.

Lemma 9. The map $\Pi_{F}$ is $\frac{1}{\sin \delta}$-Lipschitz continuous.

Proof. Given a compact convex set $\mathcal{K} \subset \mathbb{R}^{d}$, the metrical projection onto $\mathcal{K}$ is the map from $\mathbb{R}^{d}$ to $\mathcal{K}$ which associates to each point $X$ its closest point in $\mathcal{K}$. Of course this definition needs the unicity of the closest point $([6,(1.7)])$. It is well-known that the metrical projection is 1-Lipschitz (follows from [6, (11.2)]). The restriction of $\Pi_{F}$ to $\mathfrak{P}_{F} \cup \mathcal{T}_{F}$ coincides with the metrical projection onto the convex hull of $\mathfrak{P}_{F}$ and so is 1-Lipschitz.

Elementary calculus shows that the restriction of $\Pi_{F}$ to $\mathcal{Q}_{F}$ is $\frac{1}{\sin \delta}$-Lipschitz.

Now assume that $X \in \mathcal{Q}_{F}$ and $Y \in \tilde{\mathfrak{P}} \backslash \mathcal{Q}_{F}$. Let $Z$ be the intersection of $[X Y]$ with $\left[P^{\prime} B\right] \cup\left[B P^{\prime \prime}\right]$. We have

$$
\begin{aligned}
\left\|\Pi_{F}(X)-\Pi_{F}(Y)\right\| & \leq\left\|\Pi_{F}(X)-\Pi_{F}(Z)\right\|+\left\|\Pi_{F}(Z)-\Pi_{F}(Y)\right\| \\
& \leq \frac{1}{\sin \delta} X Z+Z Y \leq \frac{1}{\sin \delta}(X Z+Z Y)=\frac{1}{\sin \delta} X Y .
\end{aligned}
$$

Assume now that a sequence $\left(F_{k}\right)_{k>0}$ of finite subsets of $\mathcal{C}_{\alpha}^{\pi-\delta}$ is tending to some compact set $K \subset \overline{\mathcal{C}_{\alpha}^{\pi-\delta}}$ and put $f_{k}=\Phi_{F_{k}} \circ \Pi_{F_{k}}$. 
Lemma 10. The restriction to $K$ of $\Pi_{F_{k}}$ converges uniformly to the identity of $K$.

Proof. Just note that, for any point $X \in K$, we have

$$
\left\|\Pi_{F_{k}}(X)-X\right\| \leq d\left(X, F_{k}\right) \leq h\left(F_{k}, K\right) \rightarrow 0 .
$$

Lemma 11. For all $k, f_{k}$ is $\frac{1}{\sin \delta}$-Lipschitz; therefore the sequence $\left(f_{k}\right)_{k}$ is equicontinuous.

Proof. Let $X, Y$ be points of $\tilde{\mathfrak{P}}$ and put $x_{k}=f_{k}(X), y_{k}=f_{k}(Y)$. The $\mathbb{R}^{3}$ norm $\left\|x_{k}-y_{k}\right\|$ is less than or equal to the $\mathcal{P}_{F_{k}}$-distance between these points, which is by virtue of Lemma 6 less than the $\mathbb{R}^{2}$ norm $\left\|\Pi_{F_{k}}(X)-\Pi_{F_{k}}(Y)\right\|$. The conclusion follows from the fact that $\Pi_{F_{k}}$ is $\frac{1}{\sin \delta}$-Lipschitz.

The above lemma, together with Ascoli's well known theorem allows us to extract from $\left(f_{n}\right)$ a subsequence converging uniformly to some $\frac{1}{\sin \delta}$-Lipschitz function $f: \tilde{\mathfrak{P}} \rightarrow \mathbb{R}^{3}$. It follows that $\mathcal{S} \stackrel{\text { def }}{=} f(\mathfrak{P})$ is the limit (with respect to the Hausdorff distance) of $\left(\mathcal{P}_{F_{k}}\right)$, and therefore is a convex surface.

Lemma 12. The image by $f$ of a point of $\overline{\mathcal{C}_{\alpha}^{\pi-\delta}}$ is an antipode of o if and only if it belongs to $K \cup\left\{C_{0}, P\right\}$.

Proof. Let $X \in K \cup\left\{C_{0}, P\right\}$. Since $F_{k}$ is tending to $K$, we can chose $X_{k} \in$ $F_{k} \cup\left\{C_{0}, P\right\}$ such that the sequence $\left(X_{k}\right)$ tends to $X$. By the definition of $F_{k}$, the distance in $\mathcal{P}_{k}$ between $o$ and $f_{k}\left(X_{k}\right)$ equals 1 . Lemma 5 implies that the distance between $o$ and $f(X)$ is also 1 . On the other hand, still by virtue of Lemma 5, the distance on $\mathcal{S}$ from $o$ cannot exceed 1, hence $f(X)$ is an antipode of $o$.

Assume that $X \in \mathcal{C}_{\alpha}^{\pi-\delta}$ does not belongs to $K$, It follows that there exists a positive number $\varepsilon$, such that, for $k$ large enough, no point of $F_{k}$ lies on $\mathcal{C}_{\xi-\varepsilon}^{\xi+\varepsilon}$. Hence $X X_{k} \geq 1-\cos \varepsilon$. Moreover $\widehat{X X_{k} O}$ is obtuse, whence

$$
1=O X^{2} \geq O X_{k}^{2}+X X_{k}^{2} \geq O X_{k}^{2}+1+\cos ^{2} \varepsilon-2 \cos \varepsilon .
$$

Thus $O X_{k} \leq \sqrt{\cos \varepsilon(2-\cos \varepsilon)}<1$. Since $O X_{k}$ is also the distance between $o$ and $f_{k}(X)$ on $\mathcal{P}_{k}$, by Lemma 5 , the distance on $\mathcal{S}$ between $o$ and $f(X)$ is also less than or equal to $\sqrt{\cos \varepsilon(2-\cos \varepsilon)}$, and $f(X)$ cannot be an antipode of $o$.

Lemma 13. The set of antipodes of $o \in \mathcal{S}$ is $\left\{c_{0}, a, p\right\} \cup f(K)$.

Proof. Let $x=f(X)$ be a point of $\mathcal{S}$. As seen in the proof of Lemma 12, $x$ is an antipode of $o$ if and only if $\rho(o, x)=1$. We discuss three (overlapping) cases: 
Case 1: There are infinitely many $k \in \mathbb{N}$ such that $X \in \mathfrak{P}_{F_{k}}$. By extraction of a suitable subsequence, we can assume that $X$ belongs to $\mathfrak{P}_{F_{k}}$ for all $k$. By virtue of Lemmas 5 and 6 ,

$$
\rho(f(X), o)=\lim \rho\left(f_{k}(X), o\right)=\lim \rho\left(\Phi_{F_{k}}(X), o\right) \leq\|X\| .
$$

Hence, if $x$ is an antipode of $o$, then $X \in \overline{\mathcal{C}_{\alpha}^{2 \pi-2 \delta-\alpha}} \cup\left\{A, P^{\prime \prime}\right\}$. By Lemma 5, $a=f(A)$ and $p=f(P)=f\left(P^{\prime}\right)=f\left(P^{\prime \prime}\right)$ are antipodes of $o$. If $X$ belongs to $\overline{\mathcal{C}_{\alpha}^{\pi-\delta}}$, then the conclusion follows from Lemma 12. If $X$ belongs to $\overline{\mathcal{C}_{\pi-\delta}^{2 \pi-2 \delta-\alpha}}$, then $X$ and its image $X^{\prime}$ by the symmetry of axis $\left(O C_{0}\right)$ have the same image under $f$. Hence we can apply Lemma 5 to $X^{\prime}$ to obtain the conclusion.

Case 2: There are infinitely many $k \in \mathbb{N}$ such that $X \in \mathcal{Q}_{F_{k}}$. By extraction of a suitable subsequence, we can assume that $X$ belongs to $\mathcal{Q}_{F_{k}}$ for all $k$. By virtue of Lemmas 5 and 6 ,

$$
\rho(f(X), o)=\lim \rho\left(f_{k}(X), o\right) \leq \lim \sup \left\|\Pi_{F_{k}}(X)\right\| \leq\|X\| .
$$

Since $\lim \inf \beta$ ( $\beta$ actually depends on $k$ ) cannot vanish, by $(1),\|B\|$ (which depends on $k$ too) is less than 1 , and so $\mathcal{Q}_{F_{k}}$ does not intersect the unique circle. It follows that $f(X)$ cannot be an antipode of $o$ in this case.

Case 3: There are infinitely many $k \in \mathbb{N}$ such that $X \in \mathcal{T}_{F_{k}}$. By extraction of a suitable subsequence, we can assume that $X$ belongs to $\mathcal{T}_{F_{k}}$ for all $k$. The ray emanating from $\Pi_{F_{k}}(X)$ through $X$ intersects $\mathcal{C}_{\alpha}^{2 \pi-2 \delta-\alpha}$ at point $X_{k}$. By extraction of a suitable subsequence, we can assume that $\left(X_{k}\right)$ converges to some point $X^{\prime} \in \overline{\mathcal{C}_{\alpha}^{2 \pi-2 \delta-\alpha}}$. Since

$$
f(X)=\lim f_{k}(X)=\lim f_{k}\left(X_{k}\right)=f\left(X^{\prime}\right),
$$

the conclusion follows from Lemma 12 .

Lemma 14. The restriction to $K$ of the map $f: \tilde{\mathfrak{P}} \rightarrow \mathcal{S}$ is a local isometry, with respect to the norm of $\mathbb{R}^{2}$ and the intrinsic distance of $\mathcal{S}$.

Proof. Let $r$ be the number defined in the Lemma 8 and choose $\varepsilon \in] 0, \frac{r}{2}[$. By virtue of Lemma 10, for $k$ large enough, we have

$$
\forall X \in K\left\|\Pi_{F_{k}}(X)-X\right\|<\frac{\varepsilon}{2} .
$$

Hence, for $k$ large enough, if $X, Y \in K$ are such that $X Y \leq \frac{r}{2}<r-\varepsilon$, then, by virtue of Lemma 8,

$$
\begin{aligned}
\rho\left(f_{k}(X), f_{k}(Y)\right) & =\rho\left(\Phi_{F_{k}}\left(\Pi_{F_{k}}(X)\right), \Phi_{F_{k}}\left(\Pi_{F_{k}}(Y)\right)\right) \\
& =\left\|\Pi_{F_{k}}(X)-\Pi_{F_{k}}(Y)\right\| .
\end{aligned}
$$

On the other hand, by (5), it is clear that

$$
X Y-\varepsilon \leq\left\|\Pi_{F_{k}}(X)-\Pi_{F_{k}}(Y)\right\| \leq X Y+\varepsilon,
$$


whence, for $X Y<\frac{r}{2}$ and $k$ large enough,

$$
\left|\rho\left(f_{k}(X), f_{k}(Y)\right)-X Y\right| \leq \varepsilon
$$

Passing to the limit we find that $|\rho(f(X), f(Y))-X Y|$ is at most $\varepsilon$ for the arbitrary small positive number $\varepsilon$, and so it is zero.

Now we are in a position to prove Theorem 2 .

Proof of Theorem 2. We can assume without loss of generality that $\mathcal{H}=\mathcal{C}_{0}^{\pi}$. Let $C_{0} \in \mathcal{H}$ be the point of $K$ with the lowest abscissa, and $P$ the one with the greatest abscissa. Let $\left(F_{k}\right)_{k>0}$ be a sequence of finite subsets of $K \backslash\left\{C_{0}, P\right\}$, such that $\left(F_{k} \cup\left\{C_{0}, P\right\}\right)$ converges to $K$ with respect to the Hausdorff metric. Let $f_{k}, f$ and $\mathcal{S}$ be defined as usual. By virtue of Lemma 13, the set of antipodes of $o$ is $\{a\} \cup f(K)$. By virtue of Lemma 14, $f(K)$ is locally isometric to $K$, thus $K \cup\{U\}$ is locally isometric to $\{a\} \cup f(K)$.

Corollary 1. For any $\varepsilon>0$, there exists a convex surface $\mathcal{S}$ and a point $o \in S$ such that the length (1-dimensional Hausdorff measure) of the set of antipodes of $o$, divided by the distance between $o$ and one of its antipodes is greater than $\pi-\varepsilon$.

Proof. Apply Theorem 2 with $K=\mathcal{C}_{\varepsilon / 4}^{\pi-\varepsilon / 4}$.

Remark 4. Tudor Zamfirescu exhibited two other examples of surfaces with such large sets of antipodes [15].

Corollary 2. Any number $d \in[0,1]$ can be realized as the Hausdorff dimension of the set of antipodes of some point of some convex surface.

Proof. It is a well-known fact that the Hausdorff dimension of a Cantor set which is the disjoint union of two $\lambda$-scaled copies of itself has dimension $-\frac{\log 2}{\log \lambda}$. Consequently, if we apply Theorem 2 with a Cantor set $K$ formed of two $\left(2^{-1 / d}\right)$ scaled copies of itself, we obtain a set of antipodes of dimension $d$.

Acknowledgement. The authors thank the anonymous referee for numerous corrections and suggestions that strongly improved the paper, and Augustin Fruchard for his comments and his interest on their work.

\section{References}

[1] Pankaj K. Agarwal, Boris Aronov, Joseph O'Rourke, and Catherine A. Schevon, Star unfolding of a polytope with applications., SIAM J. Comput. 26 (1997), no. 6, 1689-1713.

[2] A. D. Alexandrov, Die innere Geometrie der konvexen Flächen., (Mathematische Lehrbücher und Monographien, II. Abt. Bd. IV.) Berlin: Akademie-Verlag. XVII, 522 S., 100 Abb. , 1955 (German. Russian original). 
[3] _ Convex polyhedra, Springer, 2005, Transl. from the Russian by N. S. Dairbekov, S. S. Kutateladze and A. B. Sossinsky.

[4] Dimitri Burago, Yuri Burago, and Sergei Ivanov, A course in metric geometry, American Mathematical Society, Providence, Rhode Island, 2001.

[5] Yu. Burago, M. Gromov, and G. Perel'man, A. D. Aleksandrov spaces with curvatures bounded below, Uspekhi Mat. Nauk 47 (1992), no. 2(284), 3-51, 222. MR MR1185284 (93m:53035)

[6] Herbert Buseman, Convex surfaces, Dover, New York, 2008, originally published in 1958 by Interscience Publishers, Inc.

[7] Hallard T. Croft, Kenneth J. Falconer, and Richard K. Guy, Unsolved problems in geometry, Springer-Verlag, New York, 1991.

[8] Joël Rouyer, On antipodes on a Riemannian manifold endowed with a generic riemanniann metric, Pac. J. Math. 212 (2003), no. 1, 187-200.

[9] _ On farthest points on Riemannian manifold homehomorphic to the 2-dimensional sphere, Rev. Roum. Math. Pures. Appl. 48 (2003), no. 1, 95103.

[10] $\_$, On antipodes on a convex polyhedron, Adv. Geom. 5 (2005), 497507.

[11] _ On antipodes on a convex polyhedron (II), Adv. Geom. (2010), to appear.

[12] Katsuhiro Shiohama, An introduction to the geometry of Alexandrov spaces, Lecture Notes Serie, Seoul National University, 1992.

[13] Costin Vîlcu, On two conjectures of Steinhaus, Geom. Dedicata 79 (2000), $267-275$.

[14] Costin Vîlcu and Tudor Zamfirescu, Symmetry and the farthest point mapping on convex surfaces, Adv. Geom. 6 (2006), no. 3, 379-387.

[15] Tudor Zamfirescu, On some questions about convex surfaces, Math. Nach. 172 (1995), 312-324.

[16] _ Farthest points on convex surfaces, Math. Z. 226 (1997), 623-630.

[17] _ Extreme points of the distance function on a convex surface, Trans. Amer. Math. Soc. 350 (1998), 1395-1406.

J. Rouyer, Laboratoire de Mathématiques, Informatique et Applications, EA 3993, Université de Haute Alsace, 4 rue des frères Lumière, 68093 Mulhouse, France, and 
Institute of mathematics "Simion Stoilow" of the Romanian academy, P.O. BOX 1764, RO-014700 Bucharest, Romania.

Email: Joel.Rouyer@ymail.com

T. Sari, Laboratoire de Mathématiques, Informatique et Applications, EA 3993, Université de Haute Alsace, 4 rue des frères Lumière, 68093 Mulhouse,

and

EPI MERE (INRIA-INRA), UMR MISTEA, INRA 2, pl. Viala, 34060 Montpellier, France.

Email: Tewfik.Sari@uha.fr 